\title{
ON THE ACCURACY OF BOOTSTRAPPING SAMPLE QUANTILES OF STRONGLY MIXING SEQUENCES
}

\author{
SHUXIA SUN
}

(Received 3 June 2005; revised 10 October 2005)

Communicated by V. Stefanov

\begin{abstract}
In this paper, we examine the rate of convergence of moving block bootstrap (MBB) approximations to the distributions of normalized sample quantiles based on strongly mixing observations. Under suitable smoothness and regularity conditions on the one-dimensional marginal distribution function, the rate of convergence of the MBB approximations to distributions of centered and scaled sample quantiles is of order $O\left(n^{-1 / 4} \log \log n\right)$.
\end{abstract}

2000 Mathematics subject classification: primary 62G09; secondary 62M10.

Keywords and phrases: weakly dependent, strictly stationary, strongly mixing, moving block bootstrap, sample quantile.

\section{Introduction}

Let $\left\{X_{i}\right\}_{i \in \mathbb{Z}}$ be a sequence of strictly stationary random variables defined on the probability space $(\Omega, \mathscr{F}, P)$ with common marginal distribution function $F$, where $\mathbb{Z} \equiv\{0, \pm 1, \pm 2, \ldots\}$ denotes the set of all integers. Let $F^{-1}$ be the corresponding quantile function, defined by

$$
F^{-1}(t)=\inf \{u: F(u) \geq t\}, \quad 0<t<1 .
$$

For a sample $X_{1}, \ldots, X_{n}, n \geq 1$, let $F_{n}$ denote the empirical distribution function, putting mass $1 / n$ on each $X_{i}$, that is,

$$
F_{n}(x)=n^{-1} \sum_{i=1}^{n} I\left(X_{i} \leq x\right), \quad x \in \mathbb{R},
$$

(C) 2007 Australian Mathematical Society $1446-7887 / 07 \$ A 2.00+0.00$ 
where $I(\cdot)$ denotes the indicator function, with $I(S)=0$ or 1 when the statement $S$ is false or true respectively. Then, $F_{n}^{-1}$ is the corresponding sample quantile function.

It is known that, under suitable mixing conditions on the process $\left\{X_{i}\right\}_{i \in \mathbb{Z}}$ and under mild smoothness conditions on the one-dimensional marginal distribution $F$, the centered and scaled $p$-th sample quantile, $Z_{n} \equiv \sqrt{n}\left(F_{n}^{-1}(p)-F^{-1}(p)\right)$, is asymptotically normal with mean zero and variance given by $\sigma_{\infty}^{2} / f^{2}\left(F^{-1}(p)\right)$, where

$$
\sigma_{\infty}^{2}=\sum_{i=-\infty}^{\infty} \operatorname{Cov}\left(I\left(X_{1} \leq F^{-1}(p)\right), I\left(X_{i+1} \leq F^{-1}(p)\right)\right)
$$

(compare Sen [24], Theorem 2.1 of Sun [26]). Thus, under dependence, the asymptotic variance of the $p$-th sample quantile not only involves the density of the random variable $X_{1}$ at the population quantile $F^{-1}(p)$, but at the same time, an infinite series of lag-covariances of the transformed sequence $\left\{I\left(X_{i} \leq F^{-1}(p)\right)\right\}_{i \in \mathbb{Z}}$. Sun [26] (compare Theorems 2.2 and 3.1) showed that, in spite of the more complicated form of the limit distribution of $Z_{n}$, the blocking mechanism of the moving block bootstrap (MBB) method provides a valid approximation to distributions of normalized sample quantiles in the almost sure sense, and the MBB estimator of the asymptotic variance is also strongly consistent.

It is also known that, under weak dependence, the accuracy of the block bootstrap approximations to the unknown sampling distributions of many regular statistics such as smooth functions of sample means, is of order $o\left(n^{-1 / 2}\right)$ (see Lahiri [16, 18], Götze and Küsch [11]). Thus, in these situations, the block bootstrap provides more accurate approximations than the classical normal approximation, which has accuracy of size $O\left(n^{-1 / 2}\right)$. However, this is not true with block bootstrap approximations to the distributions of irregular statistics such as sample quantiles. Singh [25] proved that the exact convergence rate of bootstrapping sample quantiles is of order $O\left(n^{-1 / 4}(\log \log n)^{1 / 2}\right)$. In this paper we extend Singh's result by showing that, under dependence, the rate of convergence of the MBB approximations to distributions of sample quantiles is of order $O\left(n^{-1 / 4} \log \log n\right)$.

The rest of this paper is organized as follows. We introduce some background material and state the main theorem in Section 2. Proofs are given in Section 3.

\section{Background}

We first introduce some background material. It is well known that Efron's [8] bootstrap method fails when the observations are dependent (see Singh [25]). Block bootstrap methods for dependent data have been put forward by several authors, notably by Hall [12], Carlstein [7], Künsch [15], Liu and Singh [20], Politis and Romano [22, 23], and Paparoditis and Politis [21]. See Lahiri [19] for a detailed 
account of results on bootstrap methods in the dependent case. In this paper we concentrate exclusively on the moving block bootstrap method (MBB) of Künsch [15] and Liu and Singh [20]. Here we give a brief description of the MBB method. Let $X_{1}, X_{2}, \ldots, X_{n}$ be a sample from the stationary process $\left\{X_{i}\right\}_{i \in \mathbb{Z}}$. For $\ell$, a positive integer between 1 and $n$, we define the overlapping blocks of size $\ell$ as

$$
B_{i}=\left(X_{i}, \ldots, X_{i+\ell-1}\right), \quad i=1, \ldots, n-\ell+1 .
$$

Let $B_{1}^{*}, \ldots, B_{b}^{*}$ be a random sample of blocks from $\left\{B_{1}, \ldots, B_{N}\right\}$, where $N=n-$ $\ell+1, b=\lfloor n / \ell\rfloor$, that is, $B_{1}^{*}, \ldots, B_{b}^{*}$ are independent and identically distributed as Uniform $\left\{B_{1}, \ldots, B_{N}\right\}$. Here and in the following, for any real number $x$, we denote by $\lfloor x\rfloor$ the largest integer not exceeding $x$ and by $\lceil x\rceil$ the smallest integer not less than $x$. The observations in the resampled block $B_{i}^{*}$ are denoted by $X_{(i-1) \ell+1}^{*}, \ldots, X_{i \ell}^{*}$, $1 \leq i \leq b$. Then, $X_{1}^{*}, \ldots, X_{\ell}^{*}, \ldots, X_{n_{1}}^{*}$ is the MBB sample, where $n_{1}=b \ell$. Let

$$
T_{n} \equiv t_{n}\left(X_{1}, \ldots, X_{n} ; \theta\right)
$$

be a random variable of interest that is a function of the random variables $\left\{X_{1}, \ldots, X_{n}\right\}$ and of some (possibly vector valued) population parameter $\theta$. Then, the MBB version of $T_{n}$ is defined as

$$
T_{n}^{*}=t_{n_{1}}\left(X_{1}^{*}, \ldots, X_{n_{1}}^{*} ; \hat{\theta}_{n}\right),
$$

where $\hat{\theta}_{n}$ is a suitable estimator of $\theta$ based on $\left\{X_{1}, \ldots, X_{n}\right\}$. The MBB estimator of the distribution of $T_{n}$ is the conditional distribution of $T_{n}^{*}$, given $\mathbf{X} \equiv\left\{\ldots, X_{1}, X_{2}, \ldots\right\}$. Throughout this paper, we use $P_{*}, E_{*}$, and $\operatorname{Var}_{*}$ to denote, respectively, the conditional probability, the conditional expectation, and the conditional variance, given $\mathbf{X}$. An alternative definition to the MBB version of $T_{n}$ of $(2.1)$ is given by resampling $\lceil n / \ell\rceil$ blocks from $\left\{B_{1}, \ldots, B_{N}\right\}$, and using the first $n$ out of the $\{n / \ell\rceil \ell$-many resampled values. However, the difference between these two versions is asymptotically negligible. To simplify the proofs of the main results, here we use the version given by (2.2) based on $b$ complete resampled blocks.

Next we define the MBB versions of the $p$-th sample quantile and of its centered and scaled version $Z_{n}$, for a given $p \in(0,1)$. Let $F_{n}^{*}$ denote the MBB empirical distribution function, that is, $F_{n}^{*}(x)=n_{1}^{-1} \sum_{i=1}^{n_{1}} I\left(X_{i}^{*} \leq x\right), \quad x \in \mathbb{R}$. Then, the MBB version of the sample quantile $\hat{\xi}_{n}=F_{n}^{-1}(p)$ is defined as $\xi_{n}^{*} \equiv F_{n}^{*-1}(p)$. Similarly, the MBB version of the centered and scaled sample quantile $Z_{n}=\sqrt{n}\left(\hat{\xi}_{n}-\xi_{p}\right)$ is given by

$$
Z_{n}^{*} \equiv \sqrt{n_{1}}\left(\xi_{n}^{*}-\tilde{\xi}_{n}\right)
$$

where $\xi_{p}=F^{-1}(p), \tilde{\xi}_{n}=\tilde{F}_{n}^{-1}(p)$, and $\tilde{F}_{n}(\cdot)=E_{*} F_{n}^{*}(\cdot)$. In the definition of the MBB version of $Z_{n}$, we center $\xi_{n}^{*}$ by $\tilde{\xi}_{n}$. As in the case of the sample mean (compare 
Lahiri [17]), this appears to be the analogous centering constant for the bootstrap sample quantile. Since $F_{n}^{*}$ is a valid distribution function for each set of resampled $\left\{X_{1}^{*}, \ldots, X_{n_{1}}^{*}\right\}$, the function $\tilde{F}_{n}(x) \equiv E_{*} F_{n}^{*}(x), x \in \mathbb{R}$ is also a valid distribution function. Hence, $\tilde{\xi}_{n}$ is well-defined. Let

$$
G_{n}(x)=P\left(Z_{n} \leq x\right), \quad x \in \mathbb{R},
$$

denote the distribution function of $Z_{n}$. Then, the MBB estimator of $G_{n}(x)$ is given by the conditional distribution of $Z_{n}^{*}$, that is, by

$$
\hat{G}_{n}(x)=P_{*}\left(Z_{n}^{*} \leq x\right), \quad x \in \mathbb{R} .
$$

Next, we introduce a dependence condition on the $X_{i}$ 's. Suppose that $\left\{X_{i}\right\}_{i \in \boldsymbol{Z}}$ are strictly stationary random variables defined on the probability space $(\Omega, \mathscr{F}, P)$. Let $\mathscr{F}_{m}^{n}$ denote the $\sigma$-field generated by random variables $X_{m}, X_{m+1}, \ldots, X_{n}$, $-\infty \leq m \leq n \leq \infty$. For $n \geq 1$, we define

$$
\alpha(n)=\sup _{m \in \mathbb{Z}} \sup _{A \in \mathscr{F}_{-\infty}^{m}, B \in \mathcal{F}_{m+n}^{\infty}}|P(A \cap B)-P(A) P(B)| .
$$

The sequence is called strongly mixing or $\alpha$-mixing if $\alpha(n) \rightarrow 0$ as $n \rightarrow \infty$. As a convention, we assume throughout this paper that unless otherwise specified, limits are taken as $n \rightarrow \infty$.

We conclude this section with the statement of the main result of the paper.

THEOREM 1. Suppose that the MBB block length $\ell$ satisfies $\ell=o\left(n^{1 / 2}\right)$. Suppose also that $\left\{X_{i}\right\}_{i \in \mathbb{Z}}$ is a strictly stationary $\alpha$-mixing process that satisfies the following:

(i) $F$ is twice differentiable in a neighborhood $\mathscr{N}_{p}$ of $\xi_{p}$ with derivative function $f$ such that

$$
\begin{aligned}
& 0<d_{0}=\inf \left\{f(x): x \in \mathscr{N}_{p}\right\} \leq \sup \left\{f(x): x \in \mathscr{N}_{p}\right\}=d_{1}<\infty \\
& 0<\inf \left\{\left|f^{\prime}(x)\right|: x \in \mathscr{N}_{p}\right\} \leq \sup \left\{\left|f^{\prime}(x)\right|: x \in \mathscr{N}_{p}\right\}<\infty
\end{aligned}
$$

(ii) $\sigma_{\infty}^{2}(x)$ is positive and has a bounded derivative in a neighborhood of $\xi_{p}$, say $\mathscr{N}_{p}$, where

$$
\sigma_{\infty}^{2}(x) \equiv \sum_{j=-\infty}^{\infty} \operatorname{Cov}\left(I\left(X_{1} \leq x\right), I\left(X_{1+j} \leq x\right)\right) ;
$$

(iii) there exists $d \in(0,1)$ such that $G_{i}(y) \equiv P\left(X_{i} \leq y \mid X_{j}: j \neq i\right)$ satisfies

$$
P\left(G_{i}\left(\xi_{p}\right)=1\right) \leq p-d, \quad i \in \mathbb{Z} ;
$$


(iv) there exists $\rho \in(0,1), C>0$, such that the $\alpha$-mixing coefficients satisfy

$$
\alpha(n) \leq C \rho^{n}, \quad n \geq 1 .
$$

Then, we have

$$
\begin{aligned}
\sup _{x \in \mathbb{R}} \mid & \hat{G}_{n}(x)-G_{n}(x) \mid \\
& =O\left(\ell^{-1}+\ell n^{-1 / 2}(\log \log n)^{1 / 2}+n^{-1 / 4} \log \log n\right) \quad \text { almost surely. }
\end{aligned}
$$

Thus, any block length $\ell$ satisfying $\tilde{C}_{1} n^{1 / 4}(\log \log n)^{-1} \leq \ell \leq \tilde{C}_{2} n^{1 / 4}(\log \log n)^{-1 / 4}$ with $\tilde{C}_{1}, \tilde{C}_{2}>0$, will lead to the optimal rate of convergence, $O\left(n^{-1 / 4} \log \log n\right)$.

Conditions (2.6) and (2.8) are frequently used to ensure a nondegenerate normal distribution of the $p$-th sample quantile under dependence. Condition (2.9) is a regularity condition introduced on page 73 of Sun [26] for deriving the Berry-Esseen Theorem for the sample quantiles of $\alpha$-mixing sequences. It requires that the conditional distribution of $X_{i}$ given $\left\{X_{j}: j \neq i\right\}$ has positive mass beyond $\xi_{p}$. In the independent case, $G_{i}\left(\xi_{p}\right)=P\left(X_{i} \leq \xi_{p}\right)=p$ and hence condition (2.9) holds. Condition (2.10) holds generally for linear stationary processes such as autoregressive processes (AR) and moving averages (MA), as well as for nonlinear stationary processes including autoregressive conditional heteroscedastic (ARCH) models and threshold autoregressive (TAR) models. One of the advantages of the MBB method lies in its wide applicability to very general stationary processes.

Theorem 1 indicates that, under a suitable choice of the block length, the MBB approximation to the distribution of the normalized sample quantile of $\alpha$-mixing sequence with exponentially decaying rate has accuracy of order $O\left(n^{-1 / 4} \log \log n\right)$. Thus, the MBB distribution approximation of the sample quantiles under $\alpha$-mixing dependence has approximately the same accuracy as Efron's bootstrap approximation to the distribution of samples quantiles based on independent observations. Theorem 1 is an extension of Singh's [25] result, from the independent and identically distributed case to the weakly dependence case.

It is well-known that the MBB approximation depends critically on the choice of the block length $\ell$, which is not an easy task in practical implementation. This explains partly why, in some special cases such as AR, and MA models, alternative bootstrap methods, for example, sieve methods, are often preferred (compare Bühlmann $[4,5]$ ). There exist in the literature some investigations on the choice of optimal block lengths. Bühlmann and Künsch [6] proposed a data-driven method for block length selection in variance estimation problems. Hall, Horowitz and Jing [14] proposed an empirical method for block length choice for situations where the optimal block length is known to be proportional to an explicit function of the sample size $n$. A direct application of Theorem 1 is that the theoretical result on the optimal block length 
may be used together with the empirical rule investigated by Hall, Horowitz and Jing [14] to produce confident regions for some unknown parameters for a wide range of stationary processes. In such cases, the confidence regions have asymptotic coverage error of size $O\left(n^{-1 / 4} \log \log n\right)$.

REMARK 1. Note that $\sigma_{\infty}^{2}(x)$ is well-defined for any $\alpha$-mixing process with exponential decaying coefficients. The smoothness conditions imposed on $\sigma_{\infty}^{2}(x)$ (compare (2.8)) and $f(x)$ (compare (2.7)) are used here to derive the accuracy of the MBB approximation. These conditions are not required for the consistency results (compare Theorem 2.2, Theorem 3.1 of Sun [26]).

REMARK 2. The slight order difference in $\log \log n$ terms between Singh's result and our result is due to the fact that, in the $\alpha$-mixing case, one cannot obtain a bound for the moment $E\left|\sum_{i=1}^{p} X_{i}\right|^{2}$ that is as sharp as that in the independent case. This is reflected in Lemma 3 and Lemma 4 below.

REMARK 3. In the independent and identically distributed case, smoothing techniques have been introduced to improve the performance of the bootstrap distribution approximation of sample quantiles. Hall, DiCiccio and Romano [13] showed that, if the distribution function is sufficiently smooth, smoothing appropriately can improve the accuracy of the bootstrap estimator from $O\left(n^{-1 / 4}(\log \log n)^{1 / 2}\right)$ to $O\left(n^{-1 / 2+\epsilon}\right)$, for any $\epsilon>0$. For other works in this context, we refer to Falk and Janas [9], and Falk and Reiss [10]. However, as we may see from the proof of Theorem 1 below, under dependence, smoothing may only improve the performance of MBB distribution approximation to be of order $O\left(n^{-1 / 4}(\log \log n)^{1 / 4}\right)$. As in the independent and identically distributed case, if the marginal distribution function $F$ is sufficiently smooth, kernel smoothing can improve the right-hand side of equation (3.25) below to be of order $O\left(\ell n^{-1 / 2}+n^{-1 / 2+\epsilon}\right)$, for arbitrary $\epsilon>0$. However, smoothing may not reduce the order of the right-hand side of equation (3.26) below.

\section{Proofs}

For proving the main theorem, we first present the following lemmas. The first lemma gives some asymptotic properties on the deviations of the empirical distribution function and the sample quantile. These are generalizations of the results of Babu and Singh [1].

LEMMA 1. Suppose that conditions (2.6) and (2.10) hold. Then, almost surely

(i) $\sup _{x \in \mathscr{N}_{p}}\left|F_{n}(x)-F(x)\right|=O\left(n^{-1 / 2}(\log \log n)^{1 / 2}\right)$,

(ii) $\sup _{0 \leq t \leq 1}\left|F_{n}^{-1}(t)-F^{-1}(t)\right|=O\left(n^{-1 / 2}(\log \log n)^{1 / 2}\right)$. 
PROOF. It is known that, $F\left(X_{n}\right), n \geq 1$, are uniform-[0,1] distributed random variables provided that $F$ is continuous. Here we use $E_{n}$, and $E_{n}^{-1}$ to denote, respectively, the empirical distribution function and the sample quantile function of the uniform distribution defined on $(0,1)$. Under condition (2.6), $F$ is continuous and strictly increasing on $\mathscr{N}_{p}$. Then, $F_{n} \circ F^{-1}=E_{n}$, and $F_{n}(x)=F_{n}\left(F^{-1}(t)\right), t=F(x), x \in \mathscr{N}_{p}$. Hence

$$
\begin{aligned}
\sup _{x \in \mathscr{N}_{p}}\left|F_{n}(x)-F(x)\right| & =\sup _{t \in\left\{F(x): x \in \mathscr{N}_{p}\right\}}\left|F_{n}\left(F^{-1}(t)\right)-t\right| \\
& =\sup _{t \in\left\{F(x): x \in \mathcal{N}_{p}\right\}}\left|E_{n}(t)-t\right| \\
& \leq \sup _{0 \leq t \leq 1}\left|E_{n}(t)-t\right| \\
& \leq c n^{-1 / 2}(\log \log n)^{1 / 2}, \quad \text { almost surely, }
\end{aligned}
$$

where $c$ is a positive constant. The last inequality followed from Lemma 3.4 of Babu and Singh [1]. So Lemma 1 (i) is proved. For the proof of Lemma 1 (ii), see Lemma 4.2 of Babu and Singh [1].

LEMMA 2. Under conditions of Theorem 1, we have

(i) $\sup _{x \in \mathbb{R}}\left|\tilde{F}_{n}(x)-F_{n}(x)\right|=O\left(\ell^{-1}\right)$, almost surely;

(ii) $\left|\tilde{\xi}_{n}-\xi_{p}\right|=O\left(\ell n^{-1}+n^{-1 / 2}(\log \log n)^{1 / 2}\right)$, almost surely.

PROOF. For the proof of part (i), see Lemma 2.4 (i) of Sun [26]. Here we only prove part (ii). By Lemma 2 (i), there exists a constant $C_{1}>0$ such that

$$
\sup _{x \in \mathbb{R}}\left|F_{n}(x)-\tilde{F}_{n}(x)\right| \leq C_{1} \ell n^{-1} \quad \text { almost surely. }
$$

Thus, by the definition of quantile function, we have

$$
F_{n}^{-1}\left(p-C_{1} \ell n^{-1}\right) \leq \tilde{F}_{n}^{-1}(p) \leq F_{n}^{-1}\left(p+C_{1} \ell n^{-1}\right) \text { almost surely. }
$$

By the conditions imposed on the density function, the mean value theorem, and Lemma 1,

$$
\begin{aligned}
\left|F_{n}^{-1}\left(p \pm C_{1} \ell n^{-1}\right)-\xi_{p}\right| \leq & \left.\mid F_{n}^{-1}\left(p \pm C_{1} \ell n^{-1}\right)\right)-F^{-1}\left(p \pm C_{1} \ell n^{-1}\right) \mid \\
& \left.+\mid F^{-1}\left(p \pm C_{1} \ell n^{-1}\right)\right)-F^{-1}(p) \mid \\
\leq & O\left(n^{-1 / 2}(\log \log n)^{1 / 2}\right)+\frac{1}{d_{0}} C_{1} \ell n^{-1} \\
= & O\left(\ell n^{-1}+n^{-1 / 2}(\log \log n)^{1 / 2}\right) .
\end{aligned}
$$

Hence, Lemma 2 (ii) follows from (3.2) and (3.3). 
LEMMA 3. Under conditions of Lemma 1 , there exists a $\rho_{0}>0$ such that whenever $0<F(a)<1,0<\epsilon \leq 1-F(a),|F(b)-F(a)| \leq \epsilon, 1 \leq u \leq M, H \geq 0$, and $\epsilon M^{59 / 60} \leq Q^{2} \leq \epsilon^{3 / 2} M^{13 / 10}$, we have

$$
\begin{aligned}
& P\left(\left|\sum_{i=H+1}^{H+u} x_{i}(a, b)\right|>2 \rho_{0} Q\right) \\
& \quad \leq K_{1} \exp \left\{-8 Q^{2} \epsilon^{-1} M^{-1}(\log \log \log M)^{-1}\right\}+K_{2} M^{-4},
\end{aligned}
$$

where, $x_{i}(a, b)=I\left(\min (a, b) \leq X_{i} \leq \max (a, b)\right)-|F(b)-F(a)|$.

Lemma 3 is a slight modification of Lemma 3.5 of Babu and Singh [1] by replacing $(\log M)^{-1}$ with $(\log \log \log M)^{-1}$. This exponential type of inequality is crucial to the establishment of the main result in this paper. As can be seen in the proof of Lemma 3.5 of Babu and Singh [1], the existence of the term $\log \log \log M$ is due to the second moment bound for the sum of $\alpha$-mixing random variables. One can prove this lemma easily by following the same arguments used by Babu and Singh [1] and using the fact that $F(X)$ follows a uniform $(0,1)$ distribution for any continuous random variable $X$ with smooth underlying distribution function $F$. We omit the proof here.

LEMMA 4. Under the conditions of Theorem 1, we have

$$
\sup _{|x|,|y| \leq \log n} R_{n}(x, y)=O\left(n^{-3 / 4} \log \log n\right), \quad \text { almost surely, }
$$

where

$$
\begin{aligned}
R_{n}(x, y)= & \mid F_{n}\left(\xi_{p}+\frac{x+y}{\sqrt{n}}\right)-F_{n}\left(\xi_{p}+\frac{y}{\sqrt{n}}\right) \\
& -F\left(\xi_{p}+\frac{x+y}{\sqrt{n}}\right)+F\left(\xi_{p}+\frac{y}{\sqrt{n}}\right) \mid(1+|x|)^{-1 / 2}
\end{aligned}
$$

PROOF. Lemma 4 can be proved by repeatedly applying Lemma 3, the countable subadditivity property of a probability measure and the Borel-Cantelli Lemma (compare Theorems 2.1 and 4.3 of Billingsley [2]). First we borrow the partition idea used by Singh [25] in his Lemma 3.2. By the nondecreasing property of $F_{n}(\cdot)$ and $F(\cdot)$, and the smoothness condition on $F(\cdot)$,

$$
\sup _{|x|,|y| \leq \log n} R_{n}(x, y)=L_{n}+O\left(n^{-1}\right)
$$

where

$$
L_{n}=\max \left\{\left|R_{n}(x, y)\right|: x, y=\frac{1}{n}, \frac{2}{n}, \ldots, \frac{\lfloor 1+n \log n\rfloor}{n}\right\} .
$$


Define $n_{r}=e^{\sqrt{r}}, A_{r}=\left\{n: n_{r} \leq n<n_{r+1}\right\}$, and

$$
d_{n_{1}}=\frac{\lfloor 1+n \log n\rfloor}{n}, \quad d_{n_{2}}=\frac{\lfloor 1+2 n \log n\rfloor}{n}, \quad d_{r}=\frac{\left\lfloor r \log n_{r+1}\right\rfloor}{r} .
$$

Then we have $n /(2 \sqrt{r+1}) \leq n_{r+1}-n_{r} \leq n_{r} / \sqrt{r}$, and

$$
n L_{n} \leq L_{n_{1}}+L_{n_{2}}+L(r)+L_{n} / r
$$

where

$$
\begin{aligned}
L_{n_{1}} & =\max _{1 \leq|x|,|y| \leq d_{n_{1}}}(1+|x|)^{-1 / 2}\left|\sum_{i=\left\lfloor n_{r}\right\rfloor+1}^{n} W_{i}\left(\xi_{p}+\frac{x+y}{\sqrt{n}}, \xi_{p}+\frac{y}{\sqrt{n}}\right)\right|, \\
L(r) & =\max _{1 \leq\left\{x|,| y \mid \leq d_{r}\right.}(1+|x|)^{-1 / 2}\left|\sum_{i=1}^{\left\lfloor n_{r}\right\rfloor} W_{i}\left(\xi_{p}+\frac{x+y}{\sqrt{n_{r}}}, \xi_{p}+\frac{y}{\sqrt{n_{r}}}\right)\right|, \\
L_{n_{2}} & =\max _{|z| \leq d_{n_{2}}}\left|\sum_{i=1}^{\left\lfloor n_{r}\right\rfloor} W_{i}\left(\xi_{p}+\frac{z}{\sqrt{n}}, \xi_{p}+\frac{\left\lfloor z r\left(n_{r} / n\right)^{1 / 2}\right\rfloor}{\sqrt{r n_{r}}}\right)\right|, \\
W_{i} & =I\left(\min \{a, b\} \leq X_{i} \leq \max \{a, b\}\right)-|F(b)-F(a)| .
\end{aligned}
$$

Next we use Lemma 3 to show that the three terms $L_{n_{1}}, L(r)$, and $L_{n_{2}}$ are all bounded by $O\left(n^{1 / 4} \log \log n\right)$. Without loss of generality, we may assume $|x|>0$. Let $Q=(1+|x|)^{1 / 2} n^{1 / 4} \log \log n, \epsilon=d_{1} n^{-1 / 2}|x|$, and $M=n_{r} r^{-1 / 2}$. Then,

$$
\begin{aligned}
\frac{\epsilon M^{59 / 60}}{Q^{2}} & =\frac{d_{1} n^{-1 / 2}|x|\left(n_{r} r^{-1 / 2}\right)^{59 / 60}}{(1+|x|) n^{1 / 2}(\log \log n)^{2}}=\frac{d_{1}|x|}{1+|x|} n^{-1} n_{r}^{59 / 60}(\log \log n)^{-2} \\
& \leq \frac{d_{1}|x|}{1+|x|} n_{r}^{-1 / 60}\left(\log \log n_{r}\right)^{-2}<1
\end{aligned}
$$

and, it can be easily verified that $Q^{2} /\left(\epsilon^{3 / 2} M^{13 / 10}\right) \leq 1$, which leads to

$$
\epsilon M^{59 / 60} \leq Q^{2} \leq \epsilon^{3 / 2} M^{13 / 10} .
$$

Now we apply Lemma 3 to $\left\{W_{i}\right\}$ with $Q, \epsilon$, and $M$ chosen as above and arrive at

$$
\begin{aligned}
& P\left(\left|\sum_{i=\left\{n_{r}\right\rfloor+1}^{n} W_{i}\left(\xi_{p}+\frac{x+y}{\sqrt{n}}, \xi_{p}+\frac{y}{\sqrt{n}}\right)\right|>2 \rho_{0} Q\right) \\
& \leq \leq K_{1} \exp \left\{-8 Q^{2} \epsilon^{-1} M^{-1}(\log \log \log M)^{-1}\right\}+K_{2} M^{-4} \\
&= K_{1} \exp \left\{-8 d_{1}^{-1}\left(1+|x|^{-1}\right) n(\log \log n)^{2} n_{r}^{-1} r^{1 / 2}\left(\log \log \log \left(n_{r} r^{-1 / 2}\right)\right)^{-1}\right\} \\
&+K_{2}\left(n_{r} r^{-1 / 2}\right)^{-4} \\
& \leq K_{1} \exp \left\{-8 r^{1 / 2}\left(\log \log n_{r}\right)^{2}\left(\log \log \log \left(n_{r} r^{-1 / 2}\right)\right)^{-1}\right\}+K_{2} n_{r}^{-4} r^{2} \leq O\left(n_{r}^{-4} r^{2}\right) .
\end{aligned}
$$


Then, by Theorem 2.1 of Billingsley [2] and (3.6),

$$
\begin{aligned}
& P\left(\bigcup_{n \in A_{r}}\left(L_{n_{1}}>2 \rho_{0} n^{1 / 4} \log \log n\right)\right) \\
& \quad \leq\left(n_{r+1}-n_{r}\right) d_{n_{1}}^{2} O\left(n_{r}^{-4} r^{2}\right) \leq n_{r} r^{-1 / 2}(\log n)^{2} O\left(n_{r}^{-4} r^{2}\right) \leq n_{r}^{-2} .
\end{aligned}
$$

Thus $\sum_{r=1}^{\infty} P\left(\bigcup_{n \in A_{r}}\left(L_{n_{1}}>2 \rho_{0} n^{1 / 4} \log \log n\right)\right)<\infty$, which together with BorelCantelli Lemma yields

$$
L_{n_{1}} \leq 2 \rho_{0} n^{1 / 4} \log \log n, \quad \text { almost surely. }
$$

Similarly, we may apply Lemma 3 with $Q=(1+|x|)^{1 / 2} n^{1 / 4}(\log \log n), M=n_{r}$, $\epsilon=d_{1} n_{r}^{-1 / 2}|x|$ and $Q=n^{1 / 4} \log \log n, M=n_{r}$, and $\epsilon=d_{1} n_{r}^{-1 / 2} r^{-1}$, respectively, to the sequence $\left\{W_{i}(\cdot, \cdot)\right\}$ and get

$$
\begin{aligned}
L(r) & \leq 2 \rho_{0} n^{1 / 4} \log \log n, & & \text { almost surely, } \\
L_{n_{2}} & \leq 2 \rho_{0} n^{1 / 4} \log \log n, & & \text { almost surely. }
\end{aligned}
$$

Finally, equations (3.4), (3.6), (3.9)-(3.11) lead to

$$
\sup _{|x|,|y| \leq \log n} R_{n}(x, y)=O\left(n^{-3 / 4} \log \log n\right), \quad \text { almost surely. }
$$

This completes the proof of Lemma 4.

LEMMA 5. Under the conditions of Theorem 1 , if $|x| \leq \log n$, we have

$$
\tilde{F}_{n}\left(\hat{\xi}_{n}\right)-p=x f\left(\xi_{p}\right) n^{-1 / 2}+O\left(\ell n^{-1}+(1+|x|)^{1 / 2} n^{-3 / 4} \log \log n\right),
$$

almost surely, where, $\hat{\xi}_{n}=\tilde{\xi}_{n}+x n^{-1 / 2}$.

ProOF. By Lemma 1, Lemma 2 (ii) and Lemma 4, if $|x| \leq \log n$,

$$
\begin{aligned}
\mid F_{n}\left(\hat{\xi}_{n}\right) & -F\left(\hat{\xi}_{n}\right)-F_{n}\left(\tilde{\xi}_{n}\right)+F\left(\tilde{\xi}_{n}\right) \mid \\
\leq & (1+|x|)^{1 / 2} \sup _{|x| \cdot|y| \leq \log n}\left\{(1+|x|)^{-1 / 2} R_{n}(x, y)\right\} \\
= & O\left((1+|x|)^{1 / 2} n^{-3 / 4} \log \log n\right), \quad \text { almost surely, }
\end{aligned}
$$

and

$$
\begin{aligned}
\left|\tilde{F}_{n}\left(\tilde{\xi}_{n}\right)-p\right| \leq & \left|\tilde{F}_{n}\left(\tilde{\xi}_{n}\right)-\tilde{F}_{n}\left(\tilde{\xi}_{n}-0\right)\right| \leq\left|F_{n}\left(\tilde{\xi}_{n}\right)-F_{n}\left(\tilde{\xi}_{n}-0\right)\right|+O\left(\ell n^{-1}\right) \\
\leq & \left|F_{n}\left(\tilde{\xi}_{n}\right)-F_{n}\left(\xi_{p}\right)-F\left(\tilde{\xi}_{n}\right)+p\right| \\
& +\left|F_{n}\left(\xi_{p}\right) F\left(\tilde{\xi}_{n}\right)-p-F_{n}\left(\tilde{\xi}_{n}-0\right)\right|+O\left(\ell n^{-1}\right) \\
\leq & 2 \sup _{|y| \leq \log n} R_{n}(0, y)+O\left(\ell n^{-1}\right) \\
\leq & O\left(\ell n^{-1}+n^{-3 / 4} \log \log n\right), \quad \text { almost surely. }
\end{aligned}
$$


Thus, by (3.12) and (3.13), we have, almost surely,

$$
\begin{aligned}
\tilde{F}_{n}\left(\hat{\xi}_{n}\right)-p= & {\left[\tilde{F}_{n}\left(\hat{\xi}_{n}\right)-\tilde{F}_{n}\left(\tilde{\xi}_{n}\right)\right]+\left[\tilde{F}_{n}\left(\tilde{\xi}_{n}\right)-p\right] } \\
= & {\left[F_{n}\left(\hat{\xi}_{n}\right)-F\left(\hat{\xi}_{n}\right)-F_{n}\left(\tilde{\xi}_{n}\right)+F\left(\tilde{\xi}_{n}\right)\right]+\left[F\left(\hat{\xi}_{n}\right)-F\left(\tilde{\xi}_{n}\right)\right] } \\
& +O\left(\ell n^{-1}\right)+O\left(\ell n^{-1}+n^{-3 / 4} \log \log n\right) \\
= & f\left(\epsilon_{n}\right) x n^{-1 / 2}+\left(\ell n^{-1}+(1+|x|)^{1 / 2} n^{-3 / 4} \log \log n\right) \\
= & x f\left(\xi_{p}\right) n^{-1 / 2}+O\left(\ell n^{-1}+(1+|x|)^{1 / 2} n^{-3 / 4} \log \log n\right),
\end{aligned}
$$

where $\epsilon_{n}$ is a random quantity between $\tilde{\xi}_{n}$ and $\hat{\xi}_{n}$. We used condition (2.7) in the last equation.

The next two lemmas investigate, respectively, the asymptotic propertirs of the MBB variance estimator and the tail behavior of the MBB distribution function estimation of the sample quantile. We first introduce some more notation.

$$
\begin{aligned}
U_{i}(x) & \equiv \frac{1}{\ell} \sum_{j=1}^{\ell} I\left(X_{(i-1) \ell+j} \leq x\right), \quad i=1, \ldots, b ; \\
U_{i}^{*}(x) & \equiv \frac{1}{\ell} \sum_{j=1}^{\ell} I\left(X_{(i-1) \ell+j}^{*} \leq x\right), \quad i=1, \ldots, b \\
\sigma_{n}^{2}(x) & \equiv \sum_{j=-(n-1)}^{n-1}\left(1-\frac{|j|}{n}\right) \operatorname{Cov}\left(I\left(X_{1} \leq x\right), I\left(X_{1+j} \leq x\right)\right) .
\end{aligned}
$$

We define $\hat{\xi}_{n}=\tilde{\xi}_{n}+x n^{-1 / 2}$. In the proof of Theorem 2.2 of Sun [26], it was shown that $\ell \operatorname{Var}_{*} U_{1}^{*}\left(\hat{\xi}_{n}\right) \rightarrow \sigma_{\infty}^{2}\left(\xi_{p}\right)=\sigma_{\infty}^{2}$ as $n \rightarrow \infty$. The next lemma gives a refinement.

LEMMA 6. Under the conditions of Theorem 1 , if the block length $\ell=o\left(n^{1 / 2}\right)$, then we have $\ell \operatorname{Var}_{*} U_{1}^{*}\left(\hat{\xi}_{n}\right)=\sigma_{\infty}^{2}+O\left(\ell^{-1}+\ell n^{-1 / 2}(\log \log n)^{1 / 2}\right)$ almost surely.

PROOF. Let $\sigma_{n}^{2}(x)$ and $\sigma_{\infty}^{2}(x)$ be defined as in (3.14) and (2.8), respectively. Then

$$
\begin{aligned}
\sigma_{n}^{2}(x)= & \sum_{j=-(n-1)}^{n-1}\left(1-\frac{|j|}{n}\right) \operatorname{Cov}\left(I\left(X_{1} \leq x\right), I\left(X_{1+j} \leq x\right)\right) \\
= & \sum_{j=-\infty}^{\infty} \operatorname{Cov}\left(I\left(X_{1} \leq x\right), I\left(X_{1+j} \leq x\right)\right) \\
& -\sum_{|j|=n}^{\infty} \operatorname{Cov}\left(I\left(X_{1} \leq x\right), I\left(X_{1+j} \leq x\right)\right)
\end{aligned}
$$




$$
\begin{aligned}
& -\frac{1}{n} \sum_{j=-(n-1)}^{n-1}|j| \operatorname{Cov}\left(I\left(X_{1} \leq x\right), I\left(X_{1+j} \leq x\right)\right) \\
= & \sigma_{\infty}^{2}(x)+O\left(n^{-1}\right)
\end{aligned}
$$

since, by Billingsley's inequality (see Lemma 2 of Billingsley [2, page 365])

$$
\left|\operatorname{Cov}\left(I\left(X_{1} \leq x\right), I\left(X_{1+j} \leq x\right)\right)\right| \leq 4 \cdot 1 \cdot 1 \cdot \alpha(j)=4 \alpha(j),
$$

which leads to $\sum_{|j|=n}^{\infty}\left|\operatorname{Cov}\left(I\left(X_{1} \leq x\right), I\left(X_{1+j} \leq x\right)\right)\right| \leq \sum_{|j|=n}^{\infty} 4 \alpha(j)=O\left(n^{-m}\right)$, for any $m \geq 1$, and

$$
\sum_{j=-(n-1)}^{n-1}\left|j \operatorname{Cov}\left(I\left(X_{1} \leq x\right), I\left(X_{1+j} \leq x\right)\right)\right| \leq \sum_{j=-(n-1)}^{n-1}|j| 4 \alpha(j)<\infty
$$

We next employ similar arguments used in Sun [26, Chapter 2, pages 31-33] for investigating $\ell \operatorname{Var}_{*} U_{1}^{*}\left(\hat{\xi}_{n}\right) \rightarrow \sigma_{\infty}^{2}\left(\xi_{p}\right)=\sigma_{\infty}^{2}$. By Lemma 2 , there exists constant a $\tilde{C}$ such that $\left|\tilde{\xi}_{n}-\xi_{p}\right| \leq \tilde{C} n^{-1 / 2}(\log \log n)^{1 / 2}$, almost surely. Now we let

$$
\begin{aligned}
& x_{n, 1}=\xi_{p}+x n^{-1 / 2}-\tilde{C} n^{-1 / 2}(\log \log n)^{1 / 2}, \quad \text { and } \\
& x_{n, 2}=\xi_{p}+x n^{-1 / 2}+\tilde{C} n^{-1 / 2}(\log \log n)^{1 / 2} .
\end{aligned}
$$

Then, there exists a set $A \in \mathscr{F}$ with $P(A)=1$, such that, for any $\omega \in A$, there exists a positive integer $n_{\omega}$ and for all $n>n_{\omega}$, we have

$$
x_{n, 1} \leq \hat{\xi}_{n}(\omega)=\tilde{\xi}_{n}+x n^{-1 / 2} \leq x_{n, 2}, \quad \text { almost surely }
$$

Thus, almost surely,

$$
\begin{aligned}
\operatorname{Var}_{*}\left(U_{1}^{*}\left(\hat{\xi}_{n}\right)\right)= & \frac{1}{N} \sum_{i=1}^{N} U_{i}^{2}\left(\hat{\xi}_{n}\right)-\tilde{F}_{n}^{2}\left(\hat{\xi}_{n}\right) \leq \frac{1}{N} \sum_{i=1}^{N} U_{i}^{2}\left(x_{n, 2}\right)-\tilde{F}_{n}^{2}\left(x_{n, 1}\right) \\
= & \frac{1}{N} \sum_{i=1}^{N}\left[U_{i}\left(x_{n, 2}\right)-F\left(x_{n, 2}\right)\right]^{2}+2 F\left(x_{n, 2}\right) \tilde{F}_{n}\left(x_{n, 2}\right) \\
& -F^{2}\left(x_{n, 2}\right)-\tilde{F}_{n}^{2}\left(x_{n, 1}\right),
\end{aligned}
$$

and, almost surely,

$$
\begin{aligned}
\operatorname{Var}_{*}\left(U_{1}^{*}\left(\hat{\xi}_{n}\right)\right) \geq & \frac{1}{N} \sum_{i=1}^{N} U_{i}^{2}\left(x_{n, 1}\right)-\tilde{F}_{n}^{2}\left(x_{n, 2}\right) \\
= & \frac{1}{N} \sum_{i=1}^{N}\left[U_{i}\left(x_{n, 1}\right)-F\left(x_{n, 1}\right)\right]^{2}+2 F\left(x_{n, 1}\right) \tilde{F}_{n}\left(x_{n, 1}\right) \\
& -F^{2}\left(x_{n, 1}\right)-\tilde{F}_{n}^{2}\left(x_{n, 2}\right) .
\end{aligned}
$$


By Lemmas 1 and 2 above, we have

$$
\begin{aligned}
\mid 2 F( & \left.x_{n, 2}\right) \tilde{F}_{n}\left(x_{n, 2}\right)-F^{2}\left(x_{n, 2}\right)-\tilde{F}_{n}^{2}\left(x_{n, 1}\right) \mid \\
& \leq\left|\tilde{F}_{n}^{2}\left(x_{n, 2}\right)-\tilde{F}_{n}^{2}\left(x_{n, 1}\right)\right|+\left(\tilde{F}_{n}\left(x_{n, 2}\right)-F\left(x_{n, 2}\right)\right)^{2} \\
& \leq 2\left|\tilde{F}_{n}\left(x_{n, 2}\right)-\tilde{F}_{n}\left(x_{n, 1}\right)\right|+\left(\tilde{F}_{n}\left(x_{n, 2}\right)-F\left(x_{n, 2}\right)\right)^{2} \\
& =2\left|F_{n}\left(x_{n, 2}\right)-F_{n}\left(x_{n, 1}\right)\right|+O\left(\ell^{2} n^{-2}\right), \quad \text { almost surely, }
\end{aligned}
$$

and by Lemma 4

$$
\begin{aligned}
\left|F_{n}\left(x_{n, 2}\right)-F_{n}\left(x_{n, 1}\right)\right|= & \left|\left[F_{n}\left(x_{n, 2}\right)-F_{n}\left(\xi_{p}\right)\right]-\left[F_{n}\left(x_{n, 1}\right)-F_{n}\left(\xi_{p}\right)\right]\right| \\
\leq & \left|F_{n}\left(x_{n, 2}\right)-F_{n}\left(\xi_{p}\right)-F\left(x_{n, 2}\right)+F\left(\xi_{p}\right)\right| \\
& +\left|F_{n}\left(x_{n, 1}\right)-F_{n}\left(\xi_{p}\right)-F\left(x_{n, 1}\right)+F\left(\xi_{p}\right)\right| \\
& +\left|F\left(x_{n, 2}\right)-F\left(x_{n, 1}\right)\right| \\
\leq & 2 O\left(n^{-3 / 4} \log \log n\right)+d_{1}\left|x_{n, 2}-x_{n, 1}\right| \\
= & O\left(n^{-1 / 2}(\log \log n)^{1 / 2}\right), \quad \text { almost surely. }
\end{aligned}
$$

Thus, almost surely,

$$
\ell\left|2 F\left(x_{n, 2}\right) \tilde{F}_{n}\left(x_{n, 2}\right)-F^{2}\left(x_{n, 2}\right)-\tilde{F}_{n}^{2}\left(x_{n, 1}\right)\right|=O\left(\ell n^{-1 / 2}(\log \log n)^{1 / 2}\right) .
$$

Likewise, almost surely,

$$
\ell\left|2 F\left(x_{n, 1}\right) \tilde{F}_{n}\left(x_{n, 1}\right)-F^{2}\left(x_{n, 1}\right)-\tilde{F}_{n}^{2}\left(x_{n, 2}\right)\right|=O\left(\ell n^{-1 / 2}(\log \log n)^{1 / 2}\right) .
$$

We now evaluate $\ell N^{-1} \sum_{i=1}^{N}\left[U_{i}\left(x_{n, j}\right)-F\left(x_{n, j}\right)\right]^{2}, j=1,2$. Let

$$
W_{n, i}=\ell\left[U_{i}\left(x_{n}\right)-F\left(x_{n}\right)\right]^{2}, \quad i=1, \ldots, N, \quad \text { and } \quad\left\{x_{n}\right\}=\left\{x_{n, 1}\right\},\left\{x_{n, 2}\right\} .
$$

Then

$$
\ell N^{-1} \sum_{i=1}^{N}\left[U_{i}\left(x_{n, j}\right)-F\left(x_{n, j}\right)\right]^{2}=N^{-1} \sum_{i=1}^{N} W_{n, i}, \quad j=1,2 .
$$

By (3.15) and condition (2.8) on $\sigma_{\infty}^{2}(x)$, we get, for $|x| \leq \log n$

$$
\begin{aligned}
E W_{n, i} & =E \ell\left[U_{i}\left(x_{n}\right)-F\left(x_{n}\right)\right]^{2}=\sigma_{\infty}^{2}\left(x_{n}\right)+O\left(\ell^{-1}\right) \\
& =\sigma_{\infty}^{2}\left(\xi_{p}\right)+O\left(n^{-1 / 2} \log n\right)+O\left(\ell^{-1}\right) \\
& =\sigma_{\infty}^{2}+O\left(n^{-1 / 2} \log n+\ell^{-1}\right) .
\end{aligned}
$$

Note that $w_{n, \infty}=\left\|W_{n, 1}\right\|_{\infty} \leq \ell$, where $\|\cdot\|_{\infty}$ indicates the infinity norm, and

$$
\begin{aligned}
w_{n .2}^{2} & =E W_{n, 1}^{2}=E\left(\ell\left[U_{i}\left(x_{n}\right)-F\left(x_{n}\right)\right]^{2}\right)^{2} \\
& \leq \ell E\left(\ell\left[U_{i}\left(x_{n}\right)-F\left(x_{n}\right)\right]^{2}\right) \leq C_{5} \ell, \quad \text { for some } C_{5}>0 .
\end{aligned}
$$


On the other hand, (3.21) indicates that, there exists some positive constant $C_{6}$ such that $w_{n, 2}^{2} \geq\left|E W_{n, 1}\right|^{2} \geq C_{6}$. We next apply Lemma 2.2 of Sun [26] to the triangular array $\left\{W_{n, i}\right\}$ with $a=1, q=2, d_{n}=(\log n)^{2}$, and $\epsilon_{n}=\ell^{1 / 2} n^{-1}(\log n)^{3}$, which yields

$$
\begin{aligned}
& P\left(\left|N^{-1} \sum_{i=1}^{N} W_{n, i}-E W_{n, i}\right|>\epsilon_{n}\right) \\
& \leq C^{*} \exp \left\{-\frac{C_{1}^{*}\left[n / d_{n}\right]^{2} \epsilon_{n}^{2}}{w_{n, 2}^{2}+\left[n / d_{n}\right]^{1 / 2} w_{n, \infty} \epsilon_{n}}\right\} \\
&+C^{*}\left[\frac{n}{d_{n}}\right] \max \left\{1, \frac{w_{n, 2}}{\epsilon_{n}}\right\}^{2 / 5}\left[\alpha\left(\frac{d_{n}}{2}\right)\right]^{4 / 5} \\
& \leq C^{*} \exp \left\{-\frac{C_{1}^{*} n^{2}(\log n)^{-4} \ell n^{-2}(\log n)^{6}}{C_{5} \ell+n^{1 / 2}(\log n)^{-1} \ell \cdot \ell^{1 / 2} n^{-1}(\log n)^{3}}\right\} \\
&+C^{*} n(\log n)^{-2}\left(C_{5}^{1 / 2} \ell^{1 / 2} \ell^{-1 / 2} n(\log n)^{-3}\right)^{2 / 5}\left[C \rho^{-(\log n)^{2} / 2}\right]^{4 / 5} \\
&= C^{*} \exp \left\{-\frac{C_{1}^{*}(\log n)^{2}}{C_{5}+\ell^{1 / 2} n^{-1 / 2}(\log n)^{2}}\right\} \\
&+C^{4 / 5} C^{*} C_{5}^{1 / 5} n^{7 / 5}(\log n)^{-16 / 5} \rho^{-2(\log n)^{2} / 5} \\
& \leq O\left(n^{-m}\right), \quad \text { for all } m>1 .
\end{aligned}
$$

Then, the Borel-Cantelli Lemma implies that

$$
\left|N^{-1} \sum_{i=1}^{N} W_{n, i}-E W_{n, i}\right| \leq \epsilon_{n}=\ell^{1 / 2} n^{-1}(\log n)^{3}, \quad \text { almost surely, }
$$

which together with (3.21) leads to

$$
\begin{aligned}
N^{-1} \sum_{i=1}^{N} W_{n, i} & =E W_{n, 1}+O\left(\ell^{1 / 2} n^{-1}(\log n)^{3}\right) \\
& =\sigma_{\infty}^{2}+O\left(\ell^{-1}+n^{-1 / 2} \log n\right), \quad \text { almost surely. }
\end{aligned}
$$

Hence, by (3.16)-(3.20) and (3.22), we have

$$
\begin{aligned}
\ell \operatorname{Var}_{*} U_{1}^{*}\left(\hat{\xi}_{n}\right) & =N^{-1} \sum_{i=1}^{N} W_{n, i}+O\left(\ell n^{-1 / 2}(\log \log n)^{1 / 2}\right) \\
& =\sigma_{\infty}^{2}+O\left(\ell^{-1}+n^{-1 / 2} \log n\right)+O\left(\ell n^{-1 / 2}(\log \log n)^{1 / 2}\right) \\
& =\sigma_{\infty}^{2}+O\left(\ell^{-1}+\ell n^{-1 / 2}(\log \log n)^{1 / 2}\right), \quad \text { almost surely. }
\end{aligned}
$$

Lemma 6 is proved. 
LEMMA 7. Under the conditions of Theorem 1, for any $m \geq 1$, we have, almost surely, $\left.P_{*}\left(\sqrt{n} \mid \xi_{n}^{*}-\tilde{\xi}_{n}\right) \mid>\log n\right)=O\left(n^{-m}\right)$.

PROOF. Let $\hat{y}_{n}=\tilde{\xi}_{n}+n^{-1 / 2} \log n$, then

$$
\begin{aligned}
P_{*}( & \left.\sqrt{n}\left(\xi_{n}^{*}-\tilde{\xi}_{n}\right)>\log n\right) \\
& =P_{*}\left(\xi_{n}^{*}>\tilde{\xi}_{n}+n^{-1 / 2} \log n\right) \leq P_{*}\left(F_{n}^{*}\left(\tilde{\xi}_{n}+n^{-1 / 2} \log n\right) \leq p\right) \\
& =P_{*}\left(F_{n}^{*}\left(\hat{y}_{n}\right)-\tilde{F}_{n}\left(\hat{y}_{n}\right) \leq p-\tilde{F}_{n}\left(\hat{y}_{n}\right)\right) \\
& =P_{*}\left(\frac{1}{b} \sum_{i=1}^{b}\left[U_{i}^{*}\left(\hat{y}_{n}\right)-E_{*}\left(U_{i}^{*}\left(\hat{y}_{n}\right)\right)\right] \leq p-\tilde{F}_{n}\left(\hat{y}_{n}\right)\right) .
\end{aligned}
$$

Also, by Lemma 5 , there exists $C_{0}>0$ such that $\tilde{F}_{n}\left(\hat{y}_{n}\right)-p \geq C_{0} n^{-1 / 2} \log n$, almost surely. So, we have, by (3.23), Lemma 6, and Bernstein's inequality (see Bosq [3])

$$
\begin{aligned}
P_{*}( & \left.\sqrt{n}\left(\xi_{n}^{*}-\tilde{\xi}_{n}\right)>\log n\right) \\
& \leq P_{*}\left(\sum_{i=1}^{b}\left[U_{i}^{*}\left(\hat{y}_{n}\right)-E_{*}\left(U_{i}^{*}\left(\hat{y}_{n}\right)\right)\right] \leq-C_{0} b n^{-1 / 2} \log n\right) \\
& \leq P_{*}\left(\left|\sum_{i=1}^{b}\left[\ell^{1 / 2} U_{i}^{*}\left(\hat{y}_{n}\right)-E_{*}\left(\ell^{1 / 2} U_{i}^{*}\left(\hat{y}_{n}\right)\right)\right]\right| \geq C_{0} \ell^{1 / 2} b n^{-1 / 2} \log n\right) \\
& =P_{*}\left(\left|\sum_{i=1}^{b}\left[\ell^{1 / 2} U_{i}^{*}\left(\hat{y}_{n}\right)-E_{*}\left(\ell^{1 / 2} U_{i}^{*}\left(\hat{y}_{n}\right)\right)\right]\right| \geq C_{0} b^{1 / 2} \log n\right) \\
& \leq 2 \exp \left\{-\frac{\left[C_{0} b^{1 / 2} \log n\right]^{2}}{4 \sum_{i=1}^{b} \ell E\left[U_{i}^{*}\left(\hat{y}_{n}\right)-\tilde{F}_{n}\left(\hat{y}_{n}\right)\right]^{2}+2 C_{0} b^{1 / 2} \log n}\right\} \\
& =2 \exp \left\{-\frac{C_{0}^{2} b(\log n)^{2}}{4 b \ell \operatorname{Var}_{*} U_{1}^{*}\left(\hat{y}_{n}\right)+2 C_{0} b^{1 / 2} \log n}\right\} \\
& =2 \exp \left\{-\frac{C_{0}^{2}(\log n)^{2}}{4\left(\sigma_{\infty}^{2}+o(1)\right)+2 C_{0} b^{-1 / 2} \log n}\right\} \\
& \leq O\left(n^{-m}\right), \quad \text { for all } m \geq 1, \quad \text { almost surely. }
\end{aligned}
$$

Likewise, we may show that $P_{*}\left(\sqrt{n}\left(\xi_{n}^{*}-\tilde{\xi}_{n}\right)<-\log n\right) \leq O\left(n^{-m}\right)$, for all $m \geq 1$, almost surely. This completes the proof of Lemma 7.

PROOF OF THEOREM 1. By Lemma 1 (ii) and Lemma 7, it suffices to show that almost surely

$$
\sup _{|x| \leq \log n}\left|\hat{G}_{n}(x)-G_{n}(x)\right|=O\left(\ell^{-1}+\ell n^{-1 / 2}(\log \log n)^{1 / 2}+n^{-1 / 4} \log \log n\right) .
$$


Recall that

$$
G_{n}(x)=P\left(\sqrt{n}\left(\hat{\xi}_{n}-\xi_{p}\right) \leq x\right), \quad \text { and } \quad \hat{G}_{n}(x)=P_{*}\left(\sqrt{n}\left(\xi_{n}^{*}-\tilde{\xi}_{n}\right) \leq x\right)
$$

We can show, by the Berry-Esseen Theorem for independent random variables, that

$$
\left|P_{*}\left(F_{n}^{*}\left(\hat{\xi}_{n}\right)>p\right)-\Phi\left(\frac{\sqrt{b}\left(\tilde{F}_{n}\left(\hat{\xi}_{n}\right)-p\right)}{\sqrt{\operatorname{Var}_{*}\left(U_{1}^{*}\left(\hat{\xi}_{n}\right)\right)}}\right)\right| \leq \frac{3 E_{*}\left|U_{1}^{*}\left(\hat{\xi}_{n}\right)-E_{*}\left(U_{1}^{*}\left(\hat{\xi}_{n}\right)\right)\right|^{3}}{\sqrt{b}\left(\operatorname{Var}_{*}\left(U_{1}^{*}\left(\hat{\xi}_{n}\right)\right)\right)^{3 / 2}},
$$

and by the Berry-Esseen Theorem for sample quantiles under dependence (see Theorem 4.1 of Sun [26]), that

$$
\sup _{x \in \mathbb{R}}\left|P\left(\sqrt{n}\left(\hat{\xi}_{n}-\xi_{p}\right) \leq x\right)-\Phi\left(\frac{x f\left(\xi_{p}\right)}{\sigma_{\infty}}\right)\right| \leq O\left(n^{-1 / 2}\right) .
$$

Therefore,

$$
\begin{aligned}
\sup _{|x| \leq \log n}\left|P_{*}\left(\sqrt{n}\left(\xi_{n}^{*}-\tilde{\xi}_{n}\right) \leq x\right)-P\left(\sqrt{n}\left(\hat{\xi}_{n}-\xi_{p}\right) \leq x\right)\right| \\
\leq \sup _{|x| \leq \log n}\left|\Phi\left(\frac{x f\left(\xi_{p}\right)}{\sigma_{\infty}}\right)-\Phi\left(\frac{\sqrt{b}\left(\tilde{F}_{n}\left(\hat{\xi}_{n}\right)-p\right)}{\sqrt{\operatorname{Var}_{*}\left(U_{1}^{*}\left(\hat{\xi}_{n}\right)\right)}}\right)\right| \\
\quad+\sup _{|x| \leq \log n} \frac{3 E_{*}\left|U_{1}^{*}\left(\hat{\xi}_{n}\right)-E_{*}\left(U_{1}^{*}\left(\hat{\xi}_{n}\right)\right)\right|^{3}}{\sqrt{b}\left(\operatorname{Var}_{*}\left(U_{1}^{*}\left(\hat{\xi}_{n}\right)\right)\right)^{3 / 2}}+O\left(n^{-1 / 2}\right) .
\end{aligned}
$$

We have, by Lemma 5, almost surely that

$$
\sqrt{n}\left(\tilde{F}_{n}\left(\hat{\xi}_{n}\right)-p\right)=x f\left(\xi_{p}\right)+O\left(\ell n^{-1 / 2}+(1+|x|)^{1 / 2} n^{-1 / 4} \log \log n\right),
$$

and, by Lemma 6 , almost surely that

$$
\frac{1}{\sqrt{\ell \operatorname{Var}_{*}\left(U_{1}^{*}\left(\hat{\xi}_{n}\right)\right)}}=\frac{1}{\sigma_{\infty}}+O\left(\ell^{-1}+\ell n^{-1 / 2}(\log \log n)^{1 / 2}\right) .
$$

So, (3.25) and (3.26) imply, almost surely that

$$
\text { (3.27) } \begin{aligned}
& \frac{\sqrt{b}\left(\tilde{F}_{n}\left(\hat{\xi}_{n}\right)-p\right)}{\sqrt{\operatorname{Var}_{*}\left(U_{1}^{*}\left(\hat{\xi}_{n}\right)\right)}} \\
& =\frac{x f\left(\xi_{p}\right)}{\sigma_{\infty}}+O\left(\ell^{-1}+\ell n^{-1 / 2}(\log \log n)^{1 / 2}+\sqrt{1+|x|} n^{-1 / 4} \log \log n\right) .
\end{aligned}
$$


Then, for $|x| \leq \log n$,

$$
\begin{aligned}
& \Phi\left(\frac{\sqrt{b}\left(\tilde{F}_{n}\left(\hat{\xi}_{n}\right)-p\right)}{\sqrt{\operatorname{Var}_{*}\left(U_{1}^{*}\left(\hat{\xi}_{n}\right)\right)}}\right) \\
& \quad=\Phi\left(\frac{x f\left(\xi_{p}\right)}{\sigma_{\infty}}+O\left(\ell^{-1}+\ell n^{-1 / 2}(\log \log n)^{1 / 2}+(1+|x|)^{1 / 2} n^{-1 / 4} \log \log n\right)\right) \\
& \quad=\Phi\left(\frac{x f\left(\xi_{p}\right)}{\sigma_{\infty}}\right)+O\left(\ell^{-1}+\ell n^{-1 / 2}(\log \log n)^{1 / 2}+n^{-1 / 4} \log \log n\right)
\end{aligned}
$$

Also,

$$
\begin{aligned}
\frac{n \sqrt{n}}{b^{2}} E_{*}\left|U_{1}^{*}\left(\hat{\xi}_{n}\right)-E_{*}\left(U_{1}^{*}\left(\hat{\xi}_{n}\right)\right)\right|^{3} & \leq \frac{\ell^{-1} n \sqrt{n}}{b^{2}}\left[\ell E_{*}\left|U_{1}^{*}\left(\hat{\xi}_{n}\right)-E_{*}\left(U_{1}^{*}\left(\hat{\xi}_{n}\right)\right)\right|^{2}\right] \\
& =O\left(\ell n^{-1 / 2}\right) .
\end{aligned}
$$

Thus

$$
\begin{aligned}
\frac{E_{*}\left|U_{1}^{*}\left(\hat{\xi}_{n}\right)-E_{*}\left(U_{1}^{*}\left(\hat{\xi}_{n}\right)\right)\right|^{3}}{\sqrt{b}\left(\operatorname{Var}_{*}\left(U_{1}^{*}\left(\hat{\xi}_{n}\right)\right)\right)^{3 / 2}} & =\frac{n \sqrt{n}}{b^{2}} \frac{E_{*}\left|U_{1}^{*}\left(\hat{\xi}_{n}\right)-E_{*}\left(U_{1}^{*}\left(\hat{\xi}_{n}\right)\right)\right|^{3}}{\left(\ell \operatorname{Var}_{*}\left(U_{1}^{*}\left(\hat{\xi}_{n}\right)\right)\right)^{3 / 2}} \\
& \leq O\left(\ell n^{-1 / 2}\right)
\end{aligned}
$$

Hence, by (3.24), (3.28), and (3.29), almost surely

$$
\begin{aligned}
& \sup _{|x| \leq \log n}\left|P_{*}\left(\sqrt{n}\left(\xi_{n}^{*}-\tilde{\xi}_{n}\right) \leq x\right)-P\left(\sqrt{n}\left(\hat{\xi}_{n}-\xi_{p}\right) \leq x\right)\right| \\
& \quad \leq O\left(\ell^{-1}+\ell n^{-1 / 2}(\log \log n)^{1 / 2}+n^{-1 / 4} \log \log n\right)+O\left(\ell n^{-1 / 2}\right)+O\left(n^{-1 / 2}\right) \\
& \quad=O\left(\ell^{-1}+\ell n^{-1 / 2}(\log \log n)^{1 / 2}+n^{-1 / 4} \log \log n\right),
\end{aligned}
$$

and any block length $\ell$ satisfying $\tilde{C}_{1} n^{1 / 4}(\log \log n)^{-1} \leq \tilde{C}_{2} n^{1 / 4}(\log \log n)^{-1 / 4}$ with $\tilde{C}_{1}, \tilde{C}_{2}>0$ will lead to the optimal rate $O\left(n^{-1 / 4} \log \log n\right)$. This completes the proof of Theorem 1 .

We may sharpen the right-hand side of (3.29) to be of order $O\left(\ell^{1 / 2} n^{-1 / 2}\right)$, by borrowing the same arguments as used in Lemma 6. However, the result from (3.29) is sufficient for handling our problem here.

We also point out that it is possible to extend our main result to the situation where the $\alpha$-mixing coefficients decay at a sufficiently fast polynomial rate. As can be seen, Theorem 1 is mainly built upon Lemmas 1-3 and the Berry-Esseen Theorem of Sun [26]. It should not be difficult to modify Lemmas 1 and 2 by using the exponential type of inequalities for the sums of $\alpha$-mixing random variables. The Berry-Esseen Theorem actually applies to situations where the $\alpha$-mixing coefficient $\alpha(n)$ converges to zero at a (sufficiently fast) polynomial rate. As for the possible extension of Lemma 3, one may need to modify the conditions on $M$ and $Q$ in order to get a sufficiently sharp order for the right-hand side of the inequality. 


\section{Acknowledgements}

This paper is based on part of the author's Ph.D. dissertation written under the supervision of Professor Soumendra N. Lahiri. The author is very grateful to him for his guidance and for his helpful comments on the subject of this paper. The author also thanks an anonymous referee for his comments and suggestions, which helped improve the original manuscript.

\section{References}

[1] G. J. Babu and K. Singh, 'On deviations between empirical and quantile processes for mixing random variables', J. Multivariate Anal. 8 (1978), 532-549.

[2] P. Billingsley, Probability and measure (Wiley, New York, 1995).

[3] D. Bosq, Nonparametric statistics for stochastic processes (Springer, New York, 1998).

[4] P. Bühlmann, 'Sieve bootstrap for time series', Bernoulli 3 (1997), 123-148.

[5] __ 'Sieve bootstrap with variable-length Markov chains for stationary categorical time series', J. Amer. Statist. Assoc. 97 (2002), 443-456.

[6] P. Bühlmann and H. R. Künsch, 'Block length selection in the bootstrap for time series', Comput. Statist. Data Anal. 31 (1999), 295-310.

[7] E. Carlstein, 'The use of subseries methods for estimating the variance of a general statistic from a stationary time series', Ann. Statist. 14 (1986), 1171-1179.

[8] B. Efron, 'Bootstrap methods: another look at the jackknife', Ann. Statist. 7 (1979), 1-26.

[9] M. Falk and J. Janas, 'Edgeworth expansions for studentized and prepivoted sample quantiles', Statist. Probab. Let. 14 (1992), 13-24.

[10] M. Falk and R. D. Reiss, 'Weak convergence of smoothed and nonsmoothed bootstrap quantile estimates', Ann. Probab. 17 (1989), 362-371.

[11] F. Götze and H. R. Künsch, 'Second-order correctness of the blockwise bootstrap for stationary observations', Ann. Statist. 24 (1996), 1914-1933.

[12] P. Hall, 'Resampling a coverage pattern', Stoch. Proc. Appl. 20 (1985), 231-246.

[13] P. Hall, T. J. Diciccio and J. P. Romano, 'On smoothing and the bootstrap', Ann. Statist. 17 (1989), 692-704.

[14] P. Hall, J. L. Horowitz and B. Y. Jing, 'On blocking rules for the bootstrap with dependent data', Biometrika 82 (1995), 561-574.

[15] H .R. Künsch, 'The jackknife and the bootstrap for general stationary observations', Ann. Statist. 17 (1989), 1217-1261.

[16] S. N. Lahiri, 'Second order optimality of stationary bootstrap', Statist. Probab. Lett. 11 (1991), 335-341.

[17] —_, 'Edgeworth correction by moving block bootstrap for stationary and nonstationary data', in: Exploring the limits of bootstrap (eds. R. Lepage and L. Billard) (Wiley, New York, 1992) pp. 263-270.

[18] —, 'On edgeworth expansion and moving block bootstrap for studentized $m$-estimators in multiple linear regression models', J. Multivariate Anal. 56 (1996), 42-59.

[19] - Resampling methods for dependent data (Springer, New York, 2003).

[20] R. Y. Liu and K. Singh, 'Moving blocks jackknife and bootstrap capture weak convergence', in: Exploring the limits of bootstrap (eds. R. Lepage and L. Billard) (Wiley, New York, 1992) pp. 225-248. 
[21] E. Paparoditis and D. N. Politis, 'Tapered block bootstrap', Biometrika 88 (2001), 1105-1119.

[22] D. Politis and J. P. Romano, 'A circular block resampling procedure for stationary data', in: Exploring the limits of bootstrap (eds. R. Lepage and L. Billard) (Wiley, New York, 1992) pp. 263-270.

[23] _ 'Stationary bootstrap', J. Amer. Statist. Assoc. 89 (1994), 1303-1313.

[24] P. K. Sen, 'On bahadur representation of sample quantile for sequences of $\phi$-mixing random variables', J. Multivariate Anal. 2 (1972), 77-95.

[25] K. Singh, 'On asymptotic accuracy of Efron's bootstrap', Ann. Statist. 9 (1981), 1187-1195.

[26] S. Sun, Bootstrapping the sample quantile based on weakly dependent observations (Ph.D. Thesis, Department of Statistics, Iowa State University. Ames, IA, 50011, 2004), available at: http://www. wright.edu/ shxia.sun.

Department of Mathematics and Statistics

Wright State University

Dayton, $\mathrm{OH} 45435$

USA

e-mail: shuxia.sun@wright.edu 
